\title{
Finite Element Analysis for Singularly Perturbed Advection-Diffusion Robin Boundary Values Problem*
}

\author{
Songlin Chen, Weigen Hou, Xiaohui Jiang \\ School of Mathematics and Physics, Anhui University of Technology, Ma’anshan, China \\ Email: slchen@ahut.edu.cn,wghou@ahut.edu.cn, chaodao987@163.com
}

Received November 14, 2012; revised December 16, 2012; accepted January 21, 2013

Copyright (C) 2013 Songlin Chen et al. This is an open access article distributed under the Creative Commons Attribution License, which permits unrestricted use, distribution, and reproduction in any medium, provided the original work is properly cited.

\begin{abstract}
A singularly perturbed advection-diffusion two-point Robin boundary value problem whose solution has a single boundary layer is considered. Based on the piecewise linear polynomial approximation, the finite element method is applied to the problem. Estimation of the error between solution and the finite element approximation is given in energy norm on shishkin-type mesh.
\end{abstract}

Keywords: Singular Perturbation; Advection-Diffusion; Robin BVP; Finite Element Method; Shishkin Mesh; Error Estimation

\section{Introduction}

We consider the singularly perturbed advection-diffusion Robin boundary values problem

$$
\begin{aligned}
& L_{\varepsilon} y(x) \equiv-\varepsilon y^{\prime \prime}-a(x) y^{\prime}+b(x) y=f(x), \\
& x \in I \equiv(0,1) \\
& y(0)=A, y(1)+y^{\prime}(1)=B
\end{aligned}
$$

with sufficiently smooth functions $a(x), b(x), f(x)$, and a small positive parameter $0<\varepsilon \ll 1$. We assume that $a(x)$ be decreasing monotonously, moreover

$$
a(x) \geq \alpha>0, b(x) \geq \beta>0, x \in I
$$

which guarantees the unique solvability of the problem. It is well known that there exists a boundary layer of width $O(\varepsilon)$ at $x=0$ (see [1], K.W. Chang \& F.A. Howes 1984). Standard numerical methods for singularly perturbed problem exhibit spurious error unless the layeradapted-mesh, such as Shishkin mesh, B-mesh(see [2-7]) are employed, for the solutions of singularly perturbed problem usually contain layers. The main objective of the paper is to use the method of singular perturbation to give the estimation of error between solution and the finite element approximation w.r.t. some energy norm on shishkin-type mesh.

Throughout the paper, we shall use $C$ to denote a generic positive constant that is independent of $\varepsilon$ and mesh,

*This research was supported by the National Natural Science Foundation of China (Grant No. 50975003). while it can value differently at different places, we occasionally use a subscribed one such as $C_{1}$.

\section{Properties of Solution for Continuous Problem}

In this section, some properties and bounds of the exact solution and its derivatives are deduced preliminarily.

Lemma 1 (Maximum principle) Let $\varphi \in C^{2}(\bar{I})$. If $L_{\varepsilon} \varphi(x) \geq 0$ for $x \in I, \varphi(0) \geq 0, \varphi(1)+\varphi^{\prime}(1) \geq 0$, then $\varphi(x) \geq 0$ for $x \in \bar{I}$.

Proof. Assume that there exists $x^{*} \in \bar{I}$ such that

$$
\varphi\left(x^{*}\right)=\min _{x \in I} \varphi(x)<0
$$

If $x^{*}=1$, then there holds $\varphi^{\prime}\left(x^{*}\right) \leq 0$ which results in a contradiction to $\varphi(1)+\varphi^{\prime}(1) \geq 0$; Thus $x^{*} \in I$. Since we have $\varphi^{\prime \prime}\left(x^{*}\right) \geq 0, \varphi^{\prime}\left(x^{*}\right)=0$, the differential operator on $\varphi$ at $x$ gives

$$
L_{\varepsilon} \varphi\left(x^{*}\right)=-\varepsilon \varphi^{\prime \prime}\left(x^{*}\right)-a\left(x^{*}\right) \varphi^{\prime}\left(x^{*}\right)+b\left(x^{*}\right) \varphi\left(x^{*}\right)<0
$$

which result in a contradiction to $L_{\varepsilon} \varphi(x) \geq 0$, therefore we can conclude that the minimum of $\varphi$ is non-negative.

Lemma 2 (Comparison principle) If $\varphi, \psi \in C^{2}(\bar{I})$ satisfy $L_{\varepsilon} \varphi(x) \geq\left|L_{\varepsilon} \psi(x)\right|$ for $x \in I$, and $\varphi(0) \geq \psi(0)$, $\varphi(1)+\varphi^{\prime}(1) \geq\left|\psi(1)+\psi^{\prime}(1)\right|$, then $\varphi(x) \geq|\psi(x)|$ for all $x \in \bar{I}$. 
Lemma 3 (Stability result) If $\varphi \in C^{2}(\bar{I})$, then we have

$$
|\varphi(x)| \leq C\left(|\varphi(0)|+\left|\varphi(1)+\varphi^{\prime}(1)\right|+\left|\max _{t \in I} L_{\varepsilon} \varphi(t)\right|\right)
$$

for all $x \in \bar{I}$.

The Proofs of Lemma 2 and Lemma 3 are followed essentially from Lemma 1. (See [3] Roos, Stynes and Tobiska, (1996)).

Lemma 4 Let $y(x)$ be the solution to (1) (2). then there exists a constant $C$, such that for all $x \in I$, we have the splitting

$$
y(x)=u(x)+v(x)
$$

where the regular component $u(x)$ satisfy

$$
\left|u^{(k)}\right| \leq C \text { for } k=1,2
$$

while the layer component $v(x)$ satisfy

$$
\left|v^{\prime \prime}(x)\right| \leq C \varepsilon^{-2} \exp (-\alpha x / \varepsilon) .
$$

Proof. It is known that (see [4] Kellogg 1978, Chang \& Howes 1984)

$$
\left|y^{\prime \prime}(x)\right| \leq C\left(1+\varepsilon^{-2} \exp (-\alpha x / \varepsilon)\right)
$$

We assume $\varepsilon \leq 1 / e$ spontaneously since singular perturbation.

We set $x^{*}=2 \varepsilon \alpha^{-1} \ln (1 / \varepsilon)$ such that $\exp \left(-\alpha x^{*} / \varepsilon\right)=\varepsilon^{2}$ and $u(x)=y(x)$ on $\left(x^{*}, 1-x^{*}\right)$ thus $\left|u^{\prime \prime}(x)\right| \leq C$ on $\left(x^{*}, 1-x^{*}\right)$ and then extended on $(0,1)$ with $\left|u^{\prime \prime}(x)\right| \leq C$;

Next let

$$
v(x)=\left\{\begin{array}{l}
y(x)-u(x), x \in\left[0, x^{*}\right] \\
0, x \in\left[x^{*}, 1\right]
\end{array}\right.
$$

Then considering that $1<\varepsilon^{-2} \exp (-\alpha x / \varepsilon)$ on $\left[0, x^{*}\right]$, we know that $v(x)$ satisfy

$$
\left|v^{\prime \prime}(x)\right| \leq C \varepsilon^{-2} \exp (-\alpha x / \varepsilon) \text { on } I
$$

\section{Simplification}

For simplification of the original problem, we set a transformation

$$
y(x)=u(x) \exp (-x)
$$

then Equation (1), (2) are transformed to

$$
\begin{aligned}
& -\varepsilon u^{\prime \prime}-(a(x)-2 \varepsilon) u^{\prime}+(a(x)+b(x)-\varepsilon) u \\
& =f(x) \mathrm{e}^{x}, x \in I \\
& u(0)=A, \quad u^{\prime}(1)=B e
\end{aligned}
$$

Continuing, we transform the boundary values homogeneously by

$$
w(x)=u(x)-(B e x+A)
$$

at last, the problem (1), (2) are converted to

$$
\begin{aligned}
& -\varepsilon w^{\prime \prime}-A(x) w^{\prime}+B(x) w=F(x) \\
& w(0)=0, \quad w^{\prime}(1)=0
\end{aligned}
$$

where in the $A(x), B(x), F(x)$ posses the same properties as $a(x), b(x), f(x)$, thus we just make discussion on the simplified problem below

$$
\begin{aligned}
& -\varepsilon y^{\prime \prime}-a(x) y^{\prime}+b(x) y=f(x) \\
& y(0)=0, \quad y^{\prime}(1)=0
\end{aligned}
$$

\section{The Analysis of Finite Element Approximation}

We consider the Galerkin approximation in form of Find $Y \in H_{E}^{1}(I)$ such that

$$
B(Y, v)=(f, v) \text { for } \forall v \in H_{E}^{1}(I)
$$

where $H_{E}^{1}(I) \equiv\left\{v(x) / v(0)=0, v, v^{\prime} \in L^{2}(I)\right\}$, the bilinear form

$$
B(y, v) \equiv \varepsilon\left(y^{\prime}, v^{\prime}\right)-\left(a y^{\prime}, v\right)+(b y, v)
$$

And a natural norm associated with $B(y, v)$ is chosen by

$$
\|y\|^{2}=\varepsilon\left\|y^{\prime}\right\|_{2}^{2}+\beta\|y\|_{2}^{2}
$$

wherein

$$
\|y\|_{2}^{2}=\int_{0}^{1} y(x)^{2} \mathrm{~d} x
$$

is the usual 2-norm.

It is easy to see that $B(\cdot, \cdot)$ is coercive with respect to $\|\cdot\| \|$ by the assumption of the monotony of $a(x)$ which guarantees the existence of the solution of (7) (see [8-10]). Let $N$ be an even positive integer that denotes the number of mesh intervals.

We consider the space of piecewise linear function denoted by $V \subset H_{E}^{1}(I)$ as our work space, $y^{I}(x)$ denotes the piecewise linear interpolant to $y(x)$ at some special mesh points on $I$, We'll utmost estimate the error $\|y-Y\|$.

Firstly we have

$$
\|y-Y\| \leq\left\|y-y^{I}\right\|+\left\|y^{I}-Y\right\| \|
$$

For the second term of inequality (8), we make use of the coerciveness, continuousness of $B(\cdot, \cdot)$ and the Galerkin orthogonality relation: $B\left(Y-y, y^{I}-Y\right)=0$ to obtain that

$$
\begin{aligned}
\left\|y^{I}-Y\right\|^{2} & \leq B\left(y^{I}-Y, y^{I}-Y\right)=B\left(y^{I}-y, y^{I}-Y\right) \\
& \leq \beta\left\|y^{I}-y\right\|_{2} \cdot\left\|y^{I}-Y\right\|_{2} \\
& \leq C\left\|y^{I}-y\right\| \cdot\|\cdot\| y^{I}-Y \|
\end{aligned}
$$


Thus

$$
\left\|y^{I}-Y\right\| \leq C\left\|y^{I}-y\right\| .
$$

Combined with (8), we just need to estimate the interpolation error bound $\left\|y^{I}-y\right\| \|$ below.

Lemma 5 The solution $y(x)$ of (1'), (2') and its piecewise linear interpolant $y^{I}(x)$ satisfy

$$
\begin{aligned}
& \max _{\left[x_{i-1}, x_{i}\right]}\left|y(x)-y^{I}(x)\right| \leq C\left(\int_{x_{i-1}}^{x_{i}}\left(1+\varepsilon^{-1} \mathrm{e}^{-\alpha x / 2 \varepsilon}\right) \mathrm{d} x\right)^{2} \\
& \left\|y^{\prime}(x)-y^{I^{\prime}}(x)\right\|_{2} \leq C \varepsilon^{-1 / 2} \max _{i=1, \cdots, N} \int_{x_{i-1}}^{x_{i}}\left(1+\varepsilon^{-1} \mathrm{e}^{-\alpha x / 2 \varepsilon}\right) \mathrm{d} x
\end{aligned}
$$

Proof. According to the splitting of $y(x)=u(x)+v(x)$, we have correspondingly

$$
\left|y(x)-y^{I}(x)\right| \leq\left|u(x)-u^{I}(x)\right|+\left|v(x)-v^{I}(x)\right|
$$

From Lemma 1 we have

$$
\left|u(x)-u^{I}(x)\right|=\left|\frac{u^{\prime \prime}(\xi)}{2}\left(x-x_{i-1}\right)\left(x-x_{i}\right)\right| \leq C h_{i}^{2},
$$

To obtain the estimation for singular component, we use a Taylor expansion

$$
v(x)=v\left(x_{i}\right)+v^{\prime}\left(x_{i}\right)\left(x-x_{i}\right)+\int_{x_{i}}^{x} v^{\prime \prime}(t)(x-t) \mathrm{d} t
$$

to express the error bound

$$
\begin{aligned}
\left|v^{I}(x)-v(x)\right| & \leq 2 \int_{x_{i-1}}^{x_{i}}\left|v^{\prime \prime}(t)\right|\left(t-x_{i-1}\right) \mathrm{d} t \\
& \leq 2 \int_{x_{i-1}}^{x_{i}} \varepsilon^{-2} \mathrm{e}^{-\alpha t / 2 \varepsilon}\left(t-x_{i-1}\right) \mathrm{d} t
\end{aligned}
$$

Continuously, we use the inequality involved a positive monotonically decreasing function $g$ on $[a, b]$

$$
\int_{a}^{x} g(t)(t-a) \mathrm{d} t \leq \frac{1}{2}\left(\int_{a}^{x} g^{1 / 2}(t) \mathrm{d} t\right)^{2}, x \in[a, b]
$$

Thus we have

$$
\begin{aligned}
\left|v(x)-v^{I}(x)\right| & \leq 2 \int_{x_{i-1}}^{x_{i}} \varepsilon^{-2} \mathrm{e}^{-\alpha t / 2 \varepsilon}\left(t-x_{i-1}\right) \mathrm{d} t \\
& \leq\left(\int_{x_{i-1}}^{x_{i}} \varepsilon^{-1} \mathrm{e}^{-\alpha t / 4 \varepsilon} \mathrm{d} t\right)^{2} \\
& =\frac{16}{\alpha^{2}}\left(1-\mathrm{e}^{-\frac{\alpha h_{i}}{4 \varepsilon}}\right)^{2} \mathrm{e}^{-\frac{\alpha x_{i-1}}{2 \varepsilon}}, x \in\left[x_{i-1}, x_{i}\right]
\end{aligned}
$$

Hence

$$
\begin{aligned}
& \left|y(x)-y^{I}(x)\right| \leq C\left(h_{i}^{2}+\left(1-\mathrm{e}^{-\frac{\alpha h_{i}}{4 \varepsilon}}\right)^{2} \mathrm{e}^{-\frac{\alpha x_{i-1}}{2 \varepsilon}}\right), \\
& x \in\left[x_{i-1}, x_{i}\right]
\end{aligned}
$$

For the proof of the second statement, we have

$$
\begin{aligned}
\left\|y^{\prime}(x)-y^{I^{\prime}}(x)\right\|_{2}^{2} & =\int_{0}^{1}\left(y^{\prime}(x)-y^{I^{\prime}}(x)\right)^{2} \mathrm{~d} x \\
& =\int_{0}^{1}\left(y^{I}(x)-y(x)\right) y^{\prime \prime}(x) \mathrm{d} x \\
& \leq \max _{x \in[0,1]}\left|y^{I}(x)-y(x)\right|\left(\int_{0}^{1}\left|y^{\prime \prime}(x)\right| \mathrm{d} x\right) \\
& \leq C \varepsilon_{x \in[0,1]}^{-1} \max _{x}\left|y^{I}(x)-y(x)\right| .
\end{aligned}
$$

thus, lemma 5 follows.

Theorem For $y, y^{I}, Y$ defined before, when the Shishkin mesh are applied ,we have the parameter uniform error bound in the energy norm naturally associated with the weak formulation of (1'), (2')

$$
\|y-Y\| \leq C N^{-1} \ln N
$$

Proof. Firstly, we have by triangle inequality and (9)

$$
\begin{aligned}
\|y-Y\| \leq & \left\|y-y^{I}\right\|+\left\|y^{I}-Y\right\| \leq C\left\|y-y^{I}\right\| \\
\left\|y-y^{I}\right\|^{2} & =\varepsilon\left\|\left(y-y^{I}\right)^{\prime}\right\|_{2}^{2}+\beta\left\|y-y^{I}\right\|_{2}^{2} \\
& \leq \varepsilon C \varepsilon^{-1} \max _{x \in[0,1]}\left|y(x)-y^{I}(x)\right|+\beta \int_{0}^{1}\left(y-y^{I}\right)^{2} d x \\
& \leq C \max _{x \in[0,1]}\left|y(x)-y^{I}(x)\right|+\beta \max _{x \in[0,1]}\left|y(x)-y^{I}(x)\right|^{2} \\
& \leq C_{1} \max _{x \in[0,1]}\left|y(x)-y^{I}(x)\right| \\
& \leq C_{1} \max _{\substack{\left.x \in x_{i i-1}, x_{i}\right] \\
i=1, \cdots N}}\left(h_{i}{ }^{2}+\left(1-\mathrm{e}^{-\frac{\alpha h_{i}}{4 \varepsilon}}\right)^{2} \mathrm{e}^{-\frac{\alpha x_{i-1}}{2 \varepsilon}}\right)
\end{aligned}
$$

where in $C$ 's and $C_{1}$ are stated before. thus we have

$$
\left\|y-y^{I}\right\| \leq C \max _{\substack{x \in\left[x_{i-1}, x_{i}\right] \\ i=1, \cdots N}}\left(h_{i}+\left(1-\mathrm{e}^{-\alpha h_{i} / 4 \varepsilon}\right) \mathrm{e}^{-\alpha x_{i-1} / \varepsilon}\right)
$$

Now we use the classical Shishkin mesh (see [11-13]) by setting the mesh transition parameter defined by $\tau=\min \left(\frac{1}{2}, \frac{2 \varepsilon}{\alpha} \ln N\right)$ and allocate uniformly $N / 2$ points in each of $[0, \tau]$ and $[\tau, 1]$. In practice one typically has $\tau=\frac{2 \varepsilon}{\alpha} \ln N$, we just acquiesce in this case thus

$$
\begin{aligned}
& \qquad \begin{array}{l}
h_{i}=4 \varepsilon \ln N / a N, \quad i=1, \cdots, N / 2 \\
N^{-1}<h_{i}=2(1-\tau) / N \leq 2 N^{-1}, \quad i=\frac{N}{2}+1, \cdots, N \\
\text { thus for } i=\frac{N}{2}+1, \cdots, N,
\end{array}
\end{aligned}
$$




$$
h_{i}+\left(1-\mathrm{e}^{-a h_{i} / 4 \varepsilon}\right) \mathrm{e}^{-\alpha x_{i-1} / \varepsilon} \leq C N^{-1}
$$

Also for $i=1, \cdots, N / 2$

$$
\begin{aligned}
h_{i}+\left(1-\mathrm{e}^{-\alpha h_{i} / 4 \varepsilon}\right) \mathrm{e}^{-\alpha x_{i-1} / \varepsilon} & \leq h_{i}+\left(1-\mathrm{e}^{-\alpha h_{i} / 4 \varepsilon}\right) \\
& \leq C N^{-1} \ln N .
\end{aligned}
$$

Combining the above two cases reads (10).

Remark. To obtain $L^{2}(I)$ estimation, the standard Aubin-Nitche dual verification skill may be involved.

The superconvergence phenomena on Shishkin mesh for the convection-diffusion problems can be discussed according to Z. Zhang (see $[13,14]$ ).

\section{REFERENCES}

[1] K. W. Chang and F. A. Howes, "Nonlinear Singular Perturbation Phenomena: Theory and Applications," SpringVerlag, New York, 1984.

[2] S. C. Brenner and L. R. Scott, "The Mathematical Theory of Finite Element Methods,” Springer, Berlin, 1994. http://dx.doi.org/10.1007/978-1-4757-4338-8

[3] H. G. Roos, M. Stynes and L. Tobiska, "Numerical Methods for Singularly Perturbed Differential Equations,” Springer, Berlin, 1996.

http://dx.doi.org/10.1007/978-3-662-03206-0

[4] R. B. Kellogg and A. Tsan, “Analysis of Some Difference Approximations for a Singular Perturbation Problem without Turning Points," Mathematics of Computation, Vol. 32, No. 144, 1978, pp. 1025-1039. http://dx.doi.org/10.1090/S0025-5718-1978-0483484-9

[5] F. Brezzi, L. D. Marini and A. Russo, "On the Choice of Stabilizing Subgrid for Convection-Diffusion Problems,” Computer Methods in Applied Mechanics and Engineering, Vol. 194, No. 2-5, 2005, pp. 127-148. http://dx.doi.org/10.1016/j.cma.2004.02.022

[6] T. $\operatorname{Lin} \beta$ and N. Madden, "A Finite Element Analysis of Coupled System of Singularly Perturbed Reaction-Diffusion Equations," Applied Mathematics and Computation, Vol. 148, No. 3, 2004, pp. 869-880.
http://dx.doi.org/10.1016/S0096-3003(02)00955-4

[7] M. K. Kadalbajoo, A. S. Yadaw and D. Kumar, “Comparative Study of Singularly Perturbed Two-Point BVPs via: Fitted-Mesh Finite Difference Method, B-Spline Collocation Method and Finite Element Method," Applied Mathematics and Computation, Vol. 204, No. 2, 2008, pp. 713-725. http://dx.doi.org/10.1016/j.amc.2008.07.014

[8] L. P. Franca and E. G. Dutra do Carmo, "The Galerkin Gradient Least Squares Method," Computer Methods in Applied Mechanics and Engineering, Vol. 74, No. 1, 1989, pp. 41-54. http://dx.doi.org/10.1016/0045-7825(89)90085-6

[9] F. Ilinca and J.-F. Hétu, "Galerkin Gradient LeastSquares Formulations for Transient Conduction Heat Transfer," Computer Methods in Applied Mechanics and Engineering, Vol. 191, No. 27-28, 2002, pp. 3073-3097. http://dx.doi.org/10.1016/S0045-7825(02)00242-6

[10] T. Lin $\beta$, "Layer-Adapted Meshes for Convection-Diffusion Problems," Computer Methods in Applied Mechanics and Engineering, Vol. 192, No. 9-10, 2003, pp. 10611105. http://dx.doi.org/10.1016/S0045-7825(02)00630-8

[11] M. Stynes, "Steady-State Convection-Diffusion Problems," Acta Numerica, Vol. 14, 2005, pp. 445-508. http://dx.doi.org/10.1017/S0962492904000261

[12] M. Stynes and L. Tobiska, "The SDFEM for a Convection-Diffusion Problem with a Boundary Layer: Optimal Error Analysis and Enhancement of Accuracy," SIAM Journal on Numerical Analysis, Vol. 41, No. 5, 2003, pp. 1620-1642.

[13] Z. Zhang, "Finite Element Superconvergence Approximation of One Dimensional Singularly Perturbed Problems," Numerical Methods for Partial Differential Equations, Vol. 18, No. 3, 2002, pp. 374-395. http://dx.doi.org/10.1002/num.10001

[14] Z. Zhang, "Finite Element Super-Convergence on Shishkin Mesh for 2-d Convection-Diffusion Problems," Mathematical and Computer Modelling, Vol. 72, No. 243, 2003, pp. 1147-1177. http://dx.doi.org/10.1090/S0025-5718-03-01486-8 